\title{
Lesiones intraepiteliales, inflamación y atipias escamosas cérvico-uterinas en mujeres de un municipio de Antioquia, Colombia, 2014
}

\author{
Luisa Fernanda Hernández-Ramírez* \\ Jaiberth Antonio Cardona-Arias**
}

\begin{abstract}
*Microbióloga y Bioanalista. Grupo de investigación Salud y Sostenibilidad. Universidad de Antioquia. Medellín. Antioquia. Colombia.
**Microbiólogo y Bioanalista. Mgtr en Epidemiología. Docente Escuela de Microbiología. Universidad de Antioquia. Investigador de la Facultad de Medicina. Universidad Cooperativa de Colombia. Medellín. Antioquia. Colombia.

Correspondencia: Sr. Jaiberth Antonio Cardona Arias. Dirección: calle 67 Número 53-108, Bloque 5, oficina 103. Medellín. Antioquia. Colombia. Teléfono: 2198486. Correo electrónico: jaiberthcardona@gmail.com
\end{abstract}

\section{RESUMEN}

Introducción: el cáncer cérvico-uterino es el segundo más frecuente del mundo y la enfermedad maligna más prevalente de la mujer colombiana. La tamización con la citología cérvico-vaginal ha sido una herramienta clave para disminuir su morbilidad y mortalidad. Objetivo: determinar la prevalencia de lesiones escamosas intraepiteliales, atipias de células escamosas e inflamación en mujeres de un municipio de Antioquia, Colombia, y su asociación con el régimen de afiliación en salud y la zona de residencia. Materiales y métodos: estudio descriptivo transversal en 2222 mujeres. se calculó la prevalencia de hallazgos positivos e indeterminados para lesiones premalignas e inflamación según sistema Bethesda-2001, esta fue comparada según régimen de afiliación en salud, zona de residencia y edad a través de pruebas de hipótesis y razones de prevalencia. Se analizaron los factores de confusión a través de regresión logística multivariante. Resultados: el 63,3\% habitan en zona rural y el $86,1 \%$ están afiliadas al régimen subsidiado; la prevalencia de inflamación fue $53,5 \%$, de alteraciones premalignas 1,4\% y de significado indeterminado $3 \%$. Conclusión: la prevalencia de malignidad fue mayor en adolescentes, zona rural y régimen subsidiado. El estudio permitió identificar la población de mayor riesgo para el desarrollo de cáncer cérvico-uterino del municipio, información relevante para mejorar programas de tamización y prevenir la neoplasia en la región. MÉD.UIS. 2016;29(1):29-36.

Palabras clave: Neoplasia Intraepitelial cervical. Cuello del Útero. Colombia. Prevalencia.

\section{Intraepithelial lesions, inflammation and atypical squamous uterine cervical in a municipality of Antioquia, Colombia, 2014}

\section{ABSTRACT}

Introduction: the cervical cancer is the second most common malignancy worldwide and the most prevalent of colombian women, the screening with the Pap smear has been a key tool for reducing morbidity and mortality. Objective: to determine the prevalence of squamous intraepithelial lesions, atypical squamous cell and inflammation in women of a municipality of Antioquia,Colombia, and its association with health system affiliation and area of residence. Methods: a cross sectional study in 2222 women. The prevalence of positive findings and indeterminate for premalignant lesions and inflammation were determined with Bethesda System-2001, it was compared by health system affiliation, area of residence and age through hypothesis testing and prevalence ratios. We analyzed confounders through multivariate logistic regression. Results: $63.3 \%$ live in rural areas, $\mathbf{8 6 . 1 \%}$ are affiliated to the subsidized health system, the prevalence of inflammation was $53.5 \%, 1.4 \%$ of premalignant changes and $3 \%$ of undetermined significance results. Conclusion: the prevalence of malignancy was higher in adolescents, rural and subsidized. The study identified the population most at risk for developing cervical cancer in the municipality, relevant information to improve screening and prevention programs. MÉD.UIS. 2016;29(1):29-36.

Key words: Cervical Intraepithelial Neoplasia. Cervix uteri. Colombia. Prevalence.

¿Cómo citar este artículo?: Hernández-Ramírez LF, Cardona-Arias JA. Lesiones intraepiteliales, inflamación y atipias escamosas cérvico-uterinas en mujeres de un municipio de Antioquia, Colombia, 2014. MÉD.UIS. 2016;29(1):29-36.

Artículo recibido el 10 de Junio de 2015 y aceptado para publicación el 15 de Septiembre de 2015. 


\section{INTRODUCCIÓN}

El Cáncer Cérvico-Uterino (CCU) es una neoplasia maligna originada en el cuello del útero, principalmente en la zona de transformación donde confluyen células escamosas y glandulares. Los cambios displásicos en dichas células ocasionan lesiones precancerosas conocidas como Lesiones Escamosas Intraepiteliales (LEI), que pueden evolucionar a carcinomas cervicales in situ $y$ carcinoma invasor en un periodo de 20 años'

El CCU, junto al de estómago y esófago, constituye el principal cáncer asociado con la pobreza en mujeres de todo el mundo. Su principal etiología es la infección por el Virus del Papiloma Humano (VPH), el cual es transmitido sexualmente, infecta casi al $75 \%$ de la población sexualmente activa del mundo y constituye un factor necesario pero no suficiente para el desarrollo de este cáncer ${ }^{2-4}$. Además, el VPH presenta varios mecanismos para evitar la inmunidad innata y adaptativa, como el infectar y multiplicarse en los queratinocitos, los cuales presentan un ciclo de vida corto y alejado de centros inmunes, y regular la expresión de genes de interferón ${ }^{5}$.

Este tipo de cáncer es el segundo más frecuente en las mujeres en el ámbito mundial, la primera causa de muerte en mujeres de países en desarrollo y América Latina, y es responsable del $2 \%$ del total de años de vida perdidos en mujeres jóvenes y de mediana edad ${ }^{2,6,7}$. Específicamente en Colombia, es la enfermedad maligna más frecuente de la mujer y la primera causa de mortalidad en mujeres entre los 35 y 64 años $^{8}$.

Como parte de su abordaje, la tamización con la citología cérvico-vaginal ha sido una herramienta clave para disminuir la morbilidad y la mortalidad asociada a esta entidad 9 . Se ha demostrado que la detección oportuna de lesiones precursoras en personas asintomáticas ha sido efectiva al posibilitar la reducción de la incidencia y mortalidad de CCU aproximadamente en un $75 \%$ en países desarrollados ${ }^{2,10,11}$. No obstante, a pesar de sus resultados favorables en términos de desempeño diagnóstico, sencillez y bajo costo, el impacto de la citología cérvico-vaginal ha sido menor en los países en desarrollo debido a situaciones como la mala definición de la población objeto de la tamización, aplicación inoportuna en mujeres con riesgo, problemas en la técnica de laboratorio, inadecuada comunicación de los resultados de laboratorio a la mujer y al médico tratante, obstáculos para brindar tratamiento y seguimiento adecuado, ausencia de evaluación permanente de los programas de detección y tratamiento, $0^{911}$ y ausencia de políticas sanitarias en relación al cáncer uterino.

Por otra parte, es preciso aclarar que en Colombia es más frecuente la presencia de problemas de salud en mujeres que residen en área rural, quienes presentan desventajas económicas y mayores barreras geográficas, administrativas y sociales para la prestación de servicios de salud, así como las afiliadas al régimen subsidiado en salud, dado que éste incluye a personas sin capacidad de pago o denominados "población pobre y vulnerable" Sumado a ello, en estos últimos existe mayor frecuencia de necesidades básicas insatisfechas, lo que agudiza sus problemas de salud.

Dado que el conocimiento de la epidemiología de las lesiones premalignas del cérvix es fundamental para mejorar la efectividad de los programas de reducción de $C C U$, debido a que las mujeres del oriente de Antioquia presentan una elevada frecuencia de factores de riesgo para su desarrollo, a que en la zona rural y el régimen subsidiado se presentan mayores necesidades básicas insatisfechas y problemas de acceso a salud, y que no se conocen cifras acerca de la prevalencia de este cáncer ni sus lesiones precursoras en esta región, el objetivo del presente trabajo fue determinar la prevalencia de LEl, Atipias en Células Escamosas de Significado Incierto ASCUS, e inflamación en mujeres de un municipio del oriente de Antioquia, Colombia, y su asociación con el régimen de afiliación en salud y la zona de residencia.

\section{MATERIALES y MÉTOdOS}

\section{TIPO Y POBLACIÓN DE ESTUDIO}

Es un estudio descriptivo transversal realizado en usuarias de la Empresa Social del Estado, quienes fueron atendidas en el servicio de citología cérvicovaginal de la institución en el 2012, en total se incluyeron 2222 participantes. Como principal criterio de exclusión se definió la calidad de la muestra insatisfactoria (sin células de la zona de transformación, extendidos inflamatorias o hemorrágicos, o con problemas en la fijación) y la negación o exigencia de remuneración para participar en el estudio. 


\section{RECOLECCIÓN DE LA INFORMACIÓN}

Se tomaron como principales variables del estudio la edad, zona de residencia, régimen de afiliación en salud y los hallazgos oncológicos de la citología cérvico-vaginal, interpretados según el sistema Bethesda-2001. La edad se categorizó según el Departamento Administrativo Nacional de Estadística (DANE) en adolescentes entre 10 y 19 años, adulto joven entre 20 y 44 años, adulto medio 45 a 64 años y adulto mayor con edad superior a 64 años.

\section{ANÁLISIS ESTADÍSTICO}

La descripción de la población se realizó con frecuencias absolutas, relativas y medidas de resumen. Se comparó la frecuencia de inflamación, atrofia y hallazgos oncológicos positivos e indeterminados para malignidad según grupo etario, zona de residencia y afiliación en salud a través de chi cuadrado de Pearson y exacta de Fisher; y según la edad con t Student y Anova. Se realizó la prueba Kolmogorov Smirnov para evaluar el cumplimiento del supuesto de normalidad bivariada y la prueba de Levene para el supuesto de homocedasticidad; el Anova se complementó con comparaciones múltiples de Tukey. Se calcularon razones de prevalencia con sus respectivos intervalos de confianza del 95\% para comparar la inflamación, atrofia y malignidad según la procedencia, régimen de afiliación en salud y grupo etario. Además, para evaluar la presencia o no de confusión, se evaluó la asociación entre la procedencia, el régimen de afiliación en salud y el grupo etario, y se calcularon razones de odds crudas y ajustadas, éstas últimas a través de modelos de regresión logística multivariante. En todos los análisis se tomó una significación de 0,05. Los datos se almacenaron y analizaron con los programas SPSS $20{ }^{\circledR}$ y Epidat $3.1^{\circledR}$.

\section{RESULTADOS}

La población atendida durante el año 2012 fue 2261, de las cuales 39 presentaron muestra insatisfactoria. En las 2222 incluidas, 37 presentaron histerectomía y 12 pólipos, la edad promedio fue 38 años, con rango entre 13 y 86 años, el $50 \%$ de los valores centrales de la edad estuvo entre 27 y 48 años. El grupo etario más frecuente fue el de adultos jóvenes, donde el $63,3 \%$ habitan en zona rural y $86,1 \%$ están afiliadas al régimen subsidiado. La prevalencia de inflamación fue $53,5 \%$ y de atrofia 9,9\%. En los hallazgos oncológicos el $95,6 \%$ fue negativo, la prevalencia de alteraciones premalignas fue $1,4 \%$ y de las lesiones de significado indeterminado 3\% (Ver Tabla 1).

Tabla 1. Caracterización de la población y prevalencia de hallazgos oncológicos

\begin{tabular}{|c|c|c|c|}
\hline & & $\begin{array}{c}\text { Frecuencia } \\
\text { absoluta }\end{array}$ & $\begin{array}{c}\text { Frecuencia } \\
\text { relativa }\end{array}$ \\
\hline \multirow{4}{*}{ Grupo Etario } & $\begin{array}{l}\text { Adolescentes } \\
\text { (10-19 años) }\end{array}$ & 177 & 8,0 \\
\hline & $\begin{array}{l}\text { Adulto joven } \\
\text { (20-44 años) }\end{array}$ & 1320 & 59,4 \\
\hline & $\begin{array}{l}\text { Adulto medio } \\
\text { (45-64 años) }\end{array}$ & 625 & 28,1 \\
\hline & $\begin{array}{l}\text { Adulto mayor } \\
\text { (>64 años) }\end{array}$ & 100 & 4,5 \\
\hline \multirow{2}{*}{ Procedencia } & Rural & 1406 & 63,3 \\
\hline & Urbana & 816 & 36,7 \\
\hline \multirow{2}{*}{ Régimen } & Subsidiado & 1914 & 86,1 \\
\hline & Contributivo & 308 & 13,9 \\
\hline \multirow{2}{*}{$\begin{array}{l}\text { Cambios no } \\
\text { neoplásicos }\end{array}$} & Inflamación & 1189 & 53,5 \\
\hline & Atrofia & 219 & 9,9 \\
\hline \multirow{7}{*}{$\begin{array}{l}\text { Hallazgos } \\
\text { oncológicos }\end{array}$} & Negativo & 2124 & 95,6 \\
\hline & LEI Bajo grado & 27 & 1,2 \\
\hline & LEI Alto grado & 2 & 0,1 \\
\hline & LEI-H & 2 & 0,1 \\
\hline & ASCUS & 62 & 2,8 \\
\hline & $\mathrm{ASCH}$ & 4 & 0,2 \\
\hline & AGCNOS & 1 & 0,0 \\
\hline \multirow{3}{*}{$\begin{array}{l}\text { Interpretación } \\
\text { hallazgos } \\
\text { oncológicos }\end{array}$} & Negativo & 2124 & 95,6 \\
\hline & Positivo & 31 & 1,4 \\
\hline & Indeterminado & 67 & 3,0 \\
\hline
\end{tabular}

LEI: Lesiones Escamosas Intraepiteliales. ASCUS: Atypical squamous cells of undetermined significance. ASC-H: Atypical squamous cells. AGCNOS: Atypical Glandular Cells not otherwise specified.

Fuente: autores.

La zona de residencia presentó asociación con la inflamación y atrofia; por su parte, el régimen de afiliación en salud y la edad se relacionaron con la prevalencia de malignidad, inflamación y atrofia. La prevalencia de positivos para premalignidad fue mayor en el régimen subsidiado y la inflamación en adultos jóvenes, rurales y subsidiadas (Ver Tabla 2). 
Tabla 2. Comparación de la prevalencia de los hallazgos oncológicos según edad, procedencia y régimen de afiliación en salud

\begin{tabular}{|c|c|c|c|c|c|c|c|}
\hline & \multicolumn{3}{|c|}{ Oncológico \# (\%) } & \multicolumn{2}{|c|}{ Inflamación \# (\%) } & \multicolumn{2}{|c|}{ Atrofia \# (\%) } \\
\hline Grupo etario & $\mathrm{Neg}^{\mathrm{a}}$ & Pos $^{b}$ & $\operatorname{Ind}^{\mathrm{c}}$ & No & Sí & No & Sí \\
\hline Adolescentes & $167(94,4)$ & $5(2,8)$ & $5(2,8)$ & $83(46,9)$ & $94(53,1)$ & $175(98,9)$ & $2(1,1)$ \\
\hline Adulto joven & $1264(95,8)$ & $19(1,4)$ & $37(2,8)$ & $556(42,1)$ & $764(57,9)$ & $1309(99,2)$ & $11(0,8)$ \\
\hline Adulto medio & $595(95,2)$ & $7(1,1)$ & $23(3,7)$ & $331(53,0)$ & $294(47,0)$ & $468(74,9)$ & $157(25,1)$ \\
\hline Adulto mayor & $98(98,0)$ & $0(0,0)$ & $2(2,0)$ & $63(63,0)$ & $37(37,0)$ & $51(51,0)$ & $49(49,0)$ \\
\hline Valor $\mathrm{p} \mathrm{Chi}{ }^{2}$ & \multicolumn{3}{|c|}{0,432} & \multicolumn{2}{|c|}{$0,000 * *$} & \multicolumn{2}{|c|}{$0,000 * *$} \\
\hline \multicolumn{8}{|l|}{ Procedencia } \\
\hline Rural & $1342(95,4)$ & $20(1,4)$ & $44(3,1)$ & $595(42,3)$ & $811(57,7)$ & $1284(91,3)$ & $122(8,7)$ \\
\hline Urbana & $782(95,8)$ & $11(1,3)$ & $23(2,8)$ & $438(53,7)$ & $378(46,3)$ & $719(88,1)$ & $97(11,9)$ \\
\hline Valor $\mathrm{p} \mathrm{Chi}{ }^{2}$ & \multicolumn{3}{|c|}{0,908} & \multicolumn{2}{|c|}{$0,000^{* *}$} & \multicolumn{2}{|c|}{$0,014^{*}$} \\
\hline \multicolumn{8}{|l|}{ Régimen } \\
\hline Subsidiado & $1819(95,0)$ & $31(1,6)$ & $64(3,3)$ & $809(42,3)$ & $1105(57,7)$ & $1746(91,2)$ & $168(8,8)$ \\
\hline Contributivo & $305(99,0)$ & $0(0,0)$ & $3(1,0)$ & $224(72,7)$ & $84(27,3)$ & $257(83,4)$ & $51(16,6)$ \\
\hline Valor $\mathrm{p} \mathrm{Chi}^{2}$ & \multicolumn{3}{|c|}{$0,006^{* *}$} & \multicolumn{2}{|c|}{$0,000^{* *}$} & \multicolumn{2}{|c|}{$0,000^{* *}$} \\
\hline \multicolumn{8}{|l|}{ Edad } \\
\hline Media & 38,2 & 32,1 & 39,3 & 40,1 & 36,4 & 36,0 & 58,0 \\
\hline Valor $p$ & \multicolumn{3}{|c|}{$0,045^{* *}$} & \multicolumn{2}{|c|}{$0,000^{* *^{+}}$} & \multicolumn{2}{|c|}{$0,000 * *+$} \\
\hline
\end{tabular}

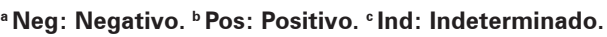

$\mathrm{Chi}^{2}$ : Prueba chi cuadrado de Pearson. ${ }^{\ddagger}$ Estadístico exacto de Fisher.

${ }^{*}$ El estadístico es significativo en el 0,05 . ${ }^{* *}$ El estadístico es significativo en el 0,01.

${ }^{\dagger}$ Prueba $\mathrm{t}$ Student para muestras independientes.

‡ Anova de una vía.

Fuente: autores.

En la edad promedio de mujeres con resultados positivos, negativos e indeterminados para malignidad, seencontróqueéstafueestadísticamente menor en los positivos frente a los negativos $(\mathrm{IC} 95 \%=0,2-12,2 ; \quad \mathrm{p}=0,044)$ e indeterminados (IC $95 \%=0,1-14,5 ; p=0,048)$, mientras que en negativos e indeterminados fue igual $(\mathrm{IC} 95 \%=-5,2-3,1 ; \mathrm{p}=0,803)$.
El grupo etario no presentó asociación estadística con el régimen de afiliación en salud, pero sí con la zona de residencia. La afiliación en salud presentó asociación con la zona de residencia, siendo mayor la afiliación al régimen subsidiado en el área rural (Ver Figura 1).

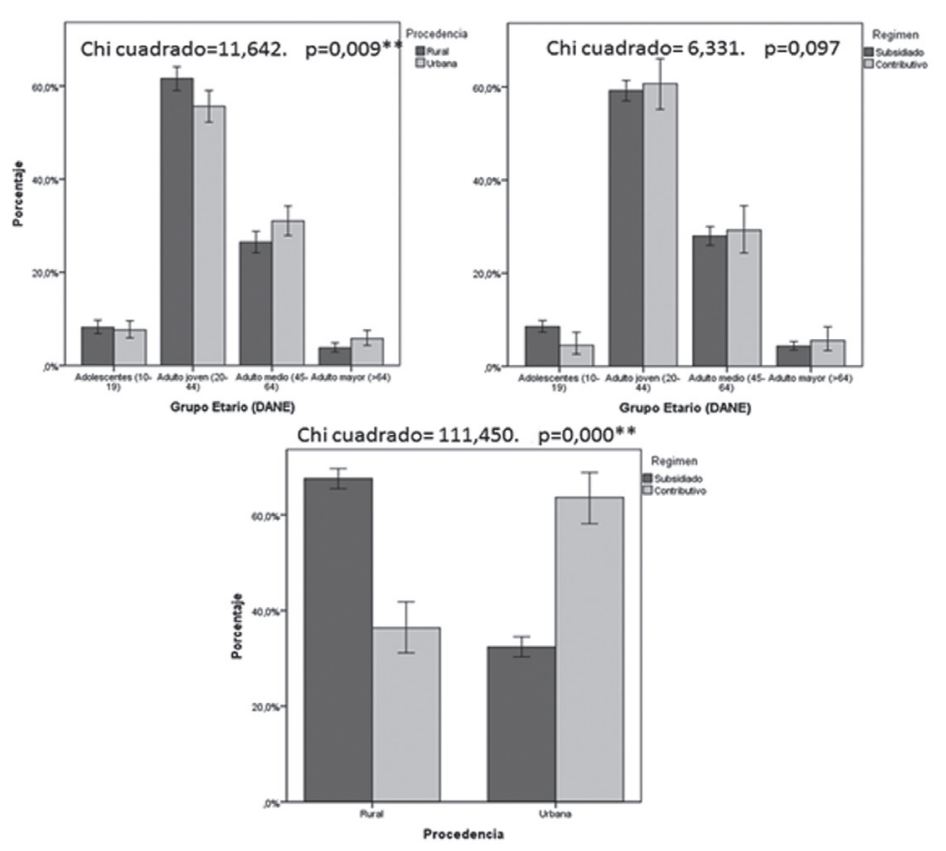

Figura 1. Asociación entre la procedencia, el régimen de afiliación en salud y el grupo etario **El estadístico es significativo en el 0,01 Fuente: autores. 


\section{ENERO-HBRIL}

La prevalencia de inflamación fue $25 \%$ mayor en las mujeres de zona rural frente a las de la zona urbana, dos veces en las afiliadas al régimen subsidiado frente al contributivo y aproximadamente 50\% mayor en las adolescentes y adultos jóvenes frente a los adultos mayores; dichas asociaciones no variaron al ajustarlas por las demás variables

Tabla 3. Medidas de asociación entre la prevalencia de malignidad, inflamación y atrofia, con algunas características de la población de estudio

\begin{tabular}{|c|c|c|c|}
\hline INFLAMACION (Sí/No) & $\begin{array}{c}\text { Razón Prevalencia } \\
\text { (IC 95\%) }\end{array}$ & $\begin{array}{l}\text { Razón de Odds (OR) } \\
\text { (IC 95\%) }\end{array}$ & $\begin{array}{l}\text { OR ajustado } \\
\text { (IC } 95 \%)\end{array}$ \\
\hline Procedencia (R/U) & $1,25(1,14-1,36)^{*}$ & $1,58(1,33-1,88)^{*}$ & $1,29(1,07-1,54)^{*}$ \\
\hline Régimen (S/C) & $2,12(1,76-2,55)^{*}$ & $3,64(2,79-4,75)^{*}$ & $3,43(2,61-4,51)^{*}$ \\
\hline Adulto mayor & 1,0 & 1,0 & \\
\hline Adolescente & $1,43(1,07-1,92)^{*}$ & $1,93(1,17-3,18)^{*}$ & $1,76(1,03-3,0)^{*}$ \\
\hline Adulto joven & $1,56(1,21-2,03)^{*}$ & $2,34(1,54-3,56)^{*}$ & $2,30(1,49-3,52)^{*}$ \\
\hline Adulto medio & $1,27(0,97-1,66)$ & $1,51(0,98-2,34)$ & $1,47(0,94-2,29)$ \\
\hline \multicolumn{4}{|l|}{ ATROFIA (Sí/No) } \\
\hline Procedencia (R/U) & $1,37(1,06-1,76)^{*}$ & $1,42(1,07-1,88)^{*}$ & $1,01(0,72-1,40)$ \\
\hline Régimen (C/S) & $1,89(1,41-2,52)^{*}$ & $2,06(1,47-2,90)^{*}$ & $2,33(1,54-3,55)^{*}$ \\
\hline Adolescente & 1,0 & 1,0 & \\
\hline Adulto joven & $0,74(0,16-3,30)$ & $0,75(0,16-3,34)$ & $0,45(0,10-2,10)$ \\
\hline Adulto medio & $22,4(5,6-89,3)^{*}$ & $29,6(7,3-120,7)^{*}$ & $17,29(4,07-73,41)^{*}$ \\
\hline Adulto mayor & $43,4(10,8-174,5)^{*}$ & $84,1(19,8-357,6)^{*}$ & $50,6(11,5-223,5)^{*}$ \\
\hline \multicolumn{4}{|l|}{ MALIGNIDAD (Positivo/Negativo) } \\
\hline Procedencia (R/U) & $1,06(0,51-2,20)$ & $1,06(0,50-2,22)$ & $0,85(0,40-1,78)$ \\
\hline Régimen $(\mathrm{S} / \mathrm{C})$ & -- & -- & -- \\
\hline Adulto medio & 1,0 & 1,0 & \\
\hline Adolescente & $2,50(0,80-7,78)$ & $2,54(0,80-8,12)$ & $2,82(0,80-9,90)$ \\
\hline Adulto joven & $1,27(0,54-3,01)$ & $1,28(0,53-3,06)$ & $1,32(0,55-3,18)$ \\
\hline \multicolumn{4}{|c|}{ MALIGNIDAD (Indeterminado/Negativo) } \\
\hline Procedencia (R/U) & $1,11(0,68-1,83)$ & $1,11(0,67-1,86)$ & $0,98(0,58-1,65)$ \\
\hline Régimen $(\mathrm{S} / \mathrm{C})$ & $3,49(1,10-11,04)^{*}$ & $3,58(1,12-11,46)^{*}$ & $3,63(1,12-11,78)^{*}$ \\
\hline Adulto mayor & 1,0 & 1,0 & \\
\hline Adolescente & $1,45(0,29-7,35)$ & $1,47(0,28-7,70)$ & $1,84(0,34-10,08)$ \\
\hline Adulto joven & $1,42(0,35-5,81)$ & $1,43(0,34-6,04)$ & $1,46(0,34-6,18)$ \\
\hline Adulto medio & $1,86(0,45-7,77)$ & $1,89(0,44-8,16)$ & $1,84(0,43-7,95)$ \\
\hline
\end{tabular}

R/U: Rural/Urbano. S/C: Subsidiado/Contributivo.

* La asociación es significativa en el 0,01.

Fuente: autores.

La prevalencia de los hallazgos no presentó asociación estadística con la zona de procedencia, la afiliación en salud ni el grupo etario; mientras que los resultados de significado indeterminado fueron tres veces mayores en el régimen subsidiado (Ver Tabla 3).

\section{Discusión}

En el actual estudio se incluyeron 2222 mujeres, esto equivale a aproximadamente al $40 \%$ de las mujeres de un municipio promedio de la región oriental independientes, descartando posibles efectos de confusión. La atrofia en las afiliadas al régimen contributivo fue dos veces la del subsidiado, y mayor en la edad adulta frente a adolescentes; según el análisis multivariado la asociación con la zona de residencia correspondía a un factor de confusión (Ver Tabla 3). 
El $86,1 \%$ estaban afiliadas al régimen subsidiado, teniendo presente que el régimen subsidiado aduce las normas de ingreso de las personas sin capacidad de pago al Sistema General de Seguridad Social en Salud, a través del pago total o parcial de la unidad de pago por capitación subsidiada con recursos fiscales o de solidaridad y afilia a "la población pobre y vulnerable"12. Se presume que los habitantes de este municipio en general y la población del actual estudio en particular, presentan desventajas económicas que ponen en riesgo su salud en la medida que presentan menos posibilidades de acceso a satisfactores de múltiples necesidades.

La prevalencia de premalignidad fue 1,4\%, inferior a lo reportado para Itagüí, Bello y el oriente antioqueño, donde fue de $2 \%, 5,9 \%$ y 7,5\%; respectivamente ${ }^{11-3}$. En el actual estudio la prevalencia de LEI presentó asociación con la edad, siendo del $2,8 \%$ en adolescentes; $1,4 \%$ en adultos jóvenes y $1,1 \%$ en adultos medio; diferente a lo observado en el estudio de Herrera, donde fue 5,9\% para menores de 20 años ${ }^{14}$. Estas diferencias evidencian la necesidad de disponer de estudios sobre la epidemiología de cada municipio o población en la que se desee implementar medidas de control, prevención y promoción.

Con respecto a la prevalencia en adolescentes se debe explicitar que la infección por VPH y sus enfermedades asociadas cada vez se presentan con mayor frecuencia en este grupo. Estudios desarrollados en Colombia indican que el 70\% de las mujeres con citología anormal tuvieron su primera relación sexual antes de los 18 años, con lo cual aumenta el riesgo de CCU por aumentar el tiempo de exposición al VPH ${ }^{4,16}$. Además, el cuello uterino es más vulnerable en esta etapa, cuando el tejido escamocolumnar sufre metaplasia y una infección con VPH podría alcanzar el tejido basal y establecer una infección persistente ${ }^{17}$.

Estudios epidemiológicos han reportado que el riesgo de neoplasia cérvico-uterina aumenta en las mujeres que inician relaciones sexuales durante la adolescencia puesto que en esa edad el cuello uterino es más susceptible a proteínas oncogénicas del $\mathrm{VPH}^{4}$. Además, en este grupo ha aumentado la prevalencia de factores de riesgo sexual como el presentar relaciones sexuales desprotegidas. Según la encuesta nacional de salud de Colombia de 2007 , la utilización del condón en la primera relación sexual fue del $18 \%$ y en la última relación sexual fue del 19\%, lo que indica que su utilización es baja y la probabilidad de adquirir una infección de transmisión sexual es muy alta.

Lo anterior podría poner de manifiesto la necesidad de implementar la vacunación contra VPH en este tipo de poblaciones, en la medida que en la actualidad se dispone de vacunas profilácticas en fase tres, con proteínas similares a la L1 de los genotipos VPH-6, 11, 16 y 18; las cuales han presentado buena seguridad y protección con elevada producción de anticuerpos contra el virus 5 .

Por otra parte, la prevalencia de premalignidad fue mayor en las afiliadas al régimen subsidiado $(1,6 \%)$ frente al contributivo (0\%), esto evidencia que la "población pobre o vulnerable" presenta mayor riesgo para el desarrollo de CCU. Ward y colaboradores indican que la población que habita en zonas de pobreza tiene $10 \%$ menos de probabilidad de supervivencia a los 5 años de habérsele diagnosticado un cáncer. Aunado a ello, las mujeres del régimen subsidiado y zona rural generalmente presentan bajo nivel educativo, el cual se ha asociado con mayor prevalencia de lesiones malignas ${ }^{18,19}$ y la norma técnica para la detección temprana del CCU y la guía de atención de lesiones pre-neoplásicas de CCU han referido que éste afecta con mayor frecuencia a las mujeres de bajo nivel socioeconómico, con multiparidad, inicio temprano de relaciones sexuales y presencia de múltiples compañeros sexuales. Todo ello evidencia la confluencia de múltiples factores que potencian el riesgo de cáncer y corrobora la necesidad del trabajo intersectorial para su control y prevención.

La prevalencia de alteraciones de significado indeterminado fue $3,7 \%$ en adultos medios, 3,3\% en el régimen subsidiado y $1 \%$ en el contributivo, esto corrobora el mayor riesgo de CCU en mujeres jóvenes y "pobres o vulnerables" y coincide con estudios previos que han reportado una menor proporción en las menores de $50^{10,20}$.

En población general, la prevalencia de ASCUS oscila entre $3,4 \%$ y $11 \%$, lo cual es compatible con lo hallado en este estudio ${ }^{15,16}$. El hallazgo citológico ASCUS no debe superar el 5\% de los resultados de tamización en población general, su importancia ha sido cuestionada por algunos investigadores que sugieren eliminarla ${ }^{10}$. Aunque los resultados de estudios de seguimiento a pacientes con esta interpretación indican que sería inapropiado hacerlo, considerando que selecciona un grupo de pacientes con mayor 


\section{ENERO-ABRIL}

riesgo de tener una LEI que el de la población general ${ }^{21}$. Esto resulta coherente con estudios que han reportado un aumento de LEI o malignidad a $46,2 \%$ y el valor predictivo positivo a $46 \%$ en mujeres con ASCUS ${ }^{10}$.

Con respecto a la inflamación, ésta presentó asociación con el grupo etario, la zona de residencia y el régimen de afiliación en salud, siendo mayor su prevalencia en adolescentes (53,1\%) y adultos jóvenes $(57,9 \%)$, zona rural $(57,7 \%)$ y régimen subsidiado (57,7\%). La importancia de la inflamación radica en que la citología inflamatoria a repetición amerita colposcopia para mejorar la evaluación; además, algunos estudios han observado que ésta persiste a pesar del tratamiento, generando anormalidades en la biopsia que fluctúan entre $20 \%$ y $70 \%$. Asociado a lo anterior, la citología inflamatoria puede estar relacionada con $\mathrm{VPH}$, donde la respuesta inmune en el huésped promueve el reclutamiento de células inflamatorias ${ }^{22}$. A diferencia de los estudios aludidos, algunos autores rechazan su utilidad como predictor de lesión premaligna para $\mathrm{CCU}^{14}$ ya que los resultados inflamatorios pueden estar asociados con procesos inespecíficos del cérvix o secundarios a infecciones como vaginosis bacteriana, Chlamydia trachomatis, Cándida albicans, Tricomonas vaginalis, virus Herpes simplex o presencia del dispositivo intrauterino ${ }^{23-6}$.

Por otra parte, es importante explicitar que la detección de lesiones precancerosas de CCU a través de la prueba de Papanicolaou ha constituido un factor clave de éxito para lograr la disminución de su mortalidad, en conjunto con algunos cambios en las prácticas sexuales, el mejoramiento en la higiene genital y la creciente frecuencia de histerectomía ${ }^{2}$. No obstante, a pesar de la disminución de la mortalidad, aún persisten retos para su control como la existencia de poca información sobre las tendencias del cáncer en los países en vía de desarrollo, segundo el aumento del consumo de tabaco en mujeres, tercero la industrialización y la urbanización, las migraciones internas y el empobrecimiento de la población que favorecen la exposición a factores de riesgo ambientales y del comportamiento que explican entre el $80 \%$ al $90 \%$ de la mortalidad por cáncer, y cuarto el hecho de que en Colombia la detección es tardía dado que los estadios superiores a II representaron $80,9 \%$ de los casos de $\mathrm{CCU}^{2}$.

Las limitaciones del estudio incluyen el sesgo temporal y la ausencia de asociaciones estadísticas causales, propias de los estudios transversales. Además, no
LESIONES ITTRAEPITELIALES, INFLAMAGIÓn Y ATIPIISS ESCAMOSAS CÉRVICO-UTERIIRS En MUJERES DE Un MUNICIPIO DE RกTIOQUIF, COLOMBIR, 2014

se realizó un muestreo probabilístico de población general, sino que los resultados corresponden a mujeres que asistieron a un servicio médico con una fuente de información limitada en el reporte de otras variables sociodemográficas y clínicas.

La reducción de la incidencia de CCU debe ser una prioridad en la agenda en salud pública del país, dado que en Colombia se encuentra en una incidencia intermedia, siendo menor que México y Venezuela, similar a la de Brasil y superior a Chile, Argentina y Puerto Rico9. Las políticas dirigidas a este problema deben ser formuladas con base en las problemáticas y los factores de riesgo particulares de cada población, para lo cual este tipo de estudio presentan un rol determinante para mejorar los programas de detección temprana, cuyo éxito radica en el reconocimiento de la población en riesgo a desarrollar CCU.

\section{CONCLUSIONES}

La prevalencia de inflamación fue elevada, al tiempo que se reportó una baja frecuencia de LEI y lesiones de significado indeterminado. La prevalencia de malignidad fue mayor en adolescentes, en zona rural y en el régimen subsidiado; con lo cual el estudio permitió identificar la población de mayor riesgo para el desarrollo de CCU del municipio. Esta información es relevante para mejorar programas de tamización y prevención de neoplasias cervical en la región.

\section{CONSIDERACIONES ÉTICAS}

En todas las etapas del proyecto se tuvieron presentes los principios de la resolución 8430 de 1993 del Ministerio de Salud de Colombia, según la cual esta investigación corresponde a un estudio sin riesgo, prevaleció la protección de los derechos y privacidad de las mujeres.

\section{CONFLICTO de INTERESES}

Los autores no manifiestan haber presentado conflicto alguno.

\section{REFERENCIAS BibLIOGRÁFICAS}

1. Dzul K, Puerto M, González M. Cáncer cervicouterino: métodos actuales para su detección. Rev Biomed. 2004;15(4):233-41.

2. Guerrero M. Neoplasias Malignas. Rev Salud Pública. 2000;2(2):173-87.

3. Burchell A, Winer R, Sanjosé S, Franco E. Epidemiology and transmission dynamics of genital HPV infection. Vaccine. 
2006;24 Suppl 3:S52-S61

4. Bosch F, Muñoz N. The viral etiology of cervical cancer. Virus Res. 2002;89(2):183-90.

5. Stanley M. Immune responses to human papillomavirus. Vaccine. 2006;24 Suppl 1:S16-S22.

6. Muñoz N. The global burden of cervical cancer in Latin America and the Caribbean: Perspectives for Prevention. Salud Pública Mex. 2007;49:29-31.

7. Yang B, Bray F, Parkin D, Sellors J, Zhang Z. Cervical cancer as a priority for prevention in different world regions: An evaluation using years of life lost. Int J Cancer. 2004;109(3):418-24.

8. Piñeros M, Ferlay J, Murillo R. Cancer incidence estimates at the national and district levels in Colombia. Salud Pública Mex. 2006;48(6):455-65.

9. Gaitán H, Rubio J, Eslava J. Asociación de la citología cervicovaginal inflamatoria con la lesión intraepitelial cervical en pacientes de una clínica de salud sexual y reproductiva en Bogotá, Colombia 1999- 2003. Rev Salud Pública. 2004;6(3):253-69.

10. Huertas S, Acosta J, Cabarcas M, Sánchez Á, Ricaurte O. Prevalencia de lesión escamosa intraepitelial (LEI) y malignidad para las atipias escamosas de significado indeterminado (ASC-US), en población perteneciente a una aseguradora pública en Colombia, 2004-2005. Rev Colomb Obstet Ginecol. 2008;59(2):124-130.

11. Hernández M, Lazcano E, De Ruiz P, Romieu I. Evaluation of the cervical cáncer screening programme in Mexico: a populationbased case control study. Int J Epidemiol. 1998;27(3):370-6.

12. Tafur L. La salud en el sistema general de seguridad social de Colombia: Régimen Subsidiado. [citado 15 de nov. 2012]. Disponible en: http://66.7.201.232/ saludput/documentos2012/ aseguramiento/FUNDAMENTOS_DEL_REGIMEN_ SUBSIDIADO EN_SALUD.pdf.

13. Alcaldía de Âbejorral. Indicadores 2012. [citado 15 de nov. 2012]. Disponible en: http://www.abejorral-antioquia.gov.co/ nuestromunicipio.shtml?apc $=$ myxx $1-\& \mathrm{~m}=\mathrm{i}$.

14. Malik S, Wilkinson E, Drew P, Hardt N. Benign cellular changes in Pap smears. Causes and significance. Acta Cytol. 2001;45(1):5-8

15. Departamento Administrativo Nacional de Estadística, Colombia Censo general 2005. Perfil Abejorral Antioquia. [citado 15 de nov. 2012]. Disponible en: http://www.dane.gov.co/files/censo2005/
PERFIL_PDF_CG2005/05002T7T000.PDF

16. Sierra C, Acosta M, Orejuela L. Papilomavirus y factores asociados a neoplasia intraepitelial cervical de alto grado en Cauca, Colombia. Rev Salud Pública. 2006; 8(Supl1):47-58.

17. Sierra C, Tyring S, Au W. Risk contribution of sexual behavior and cigarette smoking to cervical neoplasia. Int J Gynecol Cancer. 2003; 13(5):617-25

18. Tafurt Y, Acosta C, Sierra C. Prevalencia de citología anormal e infamación y su asociación con factores de riesgo para neoplasias del cuello uterino en el Cauca, Colombia. Rev. salud pública. 2012;14(1):53-66.

19. Arbalaez M, Grisales H, Vanegas A, Gaviria A, Castaño J, Mora A, et al. Prevalencia de anormalidades de células epiteliales y factores asociados en mujeres de un municipio rural colombiano. Biomédica. 2008; 28(2):271-83.

20. Flynn K, Rimm D. Diagnosis of ASC-US in women over age 50 is less likely to be associated with dysplasia. Diagn Cytopathol. 2001;24(2):132-6

21. Emerson R, Puzanov A, Brunnemer C, Younger C, Cramer H. Long-term follow-up of women with atypical squamous cells of undetermined significance (ASCUS) Diagn. Cytopathol. 2002;27(3):153-7.

22. Boccardo E, Lepique A, Villa L. The role of inflammation in HPV carcinogenesis. Carcinogenesis. 2010; 31(11):1905-12.

23. Paler R, Simpson D, Kaye A, Gunn S, Felix J. The relationship of inflammation in the papanicolaou smear to Chlamydia trachomatis infection in a high-risk population. Contraception. 2000; 61(3):231-4.

24. Franceschi S, Smith J, Van den Brule A, Herrero R, Arslan A, Anh $\mathrm{P}$, et al. Cervical infection with Chlamydia trachomatis and Neisseria gonorrhoeae in women from ten areas in fou continents. A cross-sectional study. Sex Transm Dis. 2007; 34(8):563-9.

25. Arora R, Kumar A, Prusty B, Kailash U, Batra S, Das BC Prevalence of high-risk human papillomavirus (HR-VPH) types 16 and 18 in healthy women with cytologically negative Pap smear. Eur J Obstet Gynecol Reprod Biol. 2005; 121(1):104-9.

26. Agarwal K, Sharma U, Acharya V. Microbial and cytopathological study of intrauterine contraceptive device users. Indian J Med Sci. 2004;58(9):394-9. 